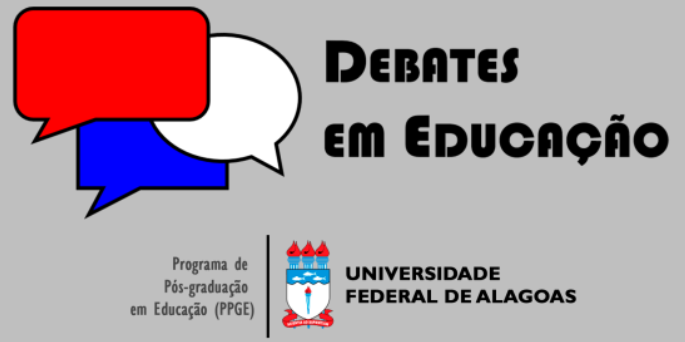

ISSN Eletrônico 2175-6600

Vol. 12 | Número Especial 2 | 2020

Joilson Ferreira de Carvalho

9 iD

Universidade do Estado de Mato Grosso (UNEMAT) joilson.carvalho@unemat.br

Daise Lago Pereira Souto

(9) ID

Universidade do Estado de Mato Grosso (UNEMAT) daise@unemat.br

William Vieira Gonçalves 9

Universidade do Estado de Mato Grosso (UNEMAT) williamvieira@unemat.br

\section{O USO DA CALCULADORA GRÁFICA GEOGEBRA EM DISPOSITIVOS MÓVEIS PARA O ENSINO DE FUNÇÕES REAIS: UM OLHAR PARA AS PUBLICAÇÕES NO BRASIL}

\section{RESUMO}

Objetivo deste artigo é apresentar um levantamento das publicações no Brasil que abordam "O uso da Calculadora Gráfica GeoGebra em dispositivos móveis para o ensino de funções reais". Para tal, foram analisadas as publicações de 2013 a 2019 , disponíveis em três bases de dados, a saber: o portal de periódicos CAPES; Scielo e o Google Acadêmico. As buscas foram feitas utilizando a combinações dos descritores "Ensino", "Função" e "GeoGebra Graphing Calculator". A metodologia utilizada foi de abordagem qualitativa e o procedimento metodológico se caracteriza como uma pesquisa bibliográfica no modelo da metassíntese qualitativa proposto por Matheus (2009). As pesquisas analisadas apontaram que a Calculadora Gráfica-GeoGebra contribuindo para o ensino aprendizagem de funções, em virtude do recurso de visualização gráfica. Com isso, estimula a participação e a cooperação dos estudantes nas aulas de matemática de modo que os mesmos sejam construtores das suas próprias aprendizagens.

Palavras-chave: Dispositivos móveis. Calculadora Gráfica GeoGebra. Ensino de funções reais.

\section{THE USE OF THE GEOGEBRA GRAPHING CALCULATOR ON MOBILE DEVICES FOR TEACHING REAL FUNCTIONS: A LOOK AT PUBLICATIONS IN BRAZIL}

\begin{abstract}
The purpose of this article is to present a survey of publications in Brazil that address "The use of the GeoGebra Graphing Calculator on mobile devices for teaching real functions". To this end, the publications from 2013 to 2019, available in three databases, were analyzed, namely: The CAPES periodical portal; Scielo and Google Scholar. The searches were made using the combinations of the descriptors "Teaching", "Function" and "GeoGebra Graphing Calculator". The methodology used has a qualitative approach and the methodological procedure is characterized as a bibliographic research in the qualitative meta-synthesis model proposed by Matheus (2009). The researches analyzed pointed out that the Graphical-GeoGebra Calculator contributes to the teaching of learning functions, due to the feature of graphical visualization. With that, it encourages the participation and cooperation of students in mathematics classes so that they are builders of their own learning.
\end{abstract}

Keywords: Mobile devices. GeoGebra graphing calculator. Teaching real functions.

Submetido em: 23/06/2020

Aceito em: 20/08/2020

Publicado em: 30/12/2020

do: http://dx.doi.org//0.28998/2175-6600.2020v12nEsp2p315-327 


\section{INTRODUÇÃO}

Atualmente, o uso de dispositivos móveis no ambiente escolar está crescendo exponencialmente. No entanto, o grande desafio da atualidade consiste no aproveitamento das suas funcionalidades no processo de ensino aprendizagem, (LIMA, FALKEMBACH, TAROUCO, 20I4).

Para a UNESCO (2014), o uso de dispositivos móveis de maneira pedagógica, contribui para a expansão e o alcance da equidade da educação, permite a aprendizagem em qualquer hora, em qualquer lugar, assegura o uso produtivo do tempo em sala de aula, permite uma ponte entre a aprendizagem formal e informal, minimiza a interrupção educacional em áreas de conflitos e desastres naturais, auxilia estudantes com deficiências e maximiza a relação custo-eficiência da educação.

Além dos dispositivos móveis, segundo Pacheco e Barros (2013), os softwares educacionais constituem-se importantes recursos pedagógicos para o processo de ensino aprendizagem. $\bigcirc$ uso consciente desses recursos possibilita a dinamização no ensino e a motivação pela aprendizagem da matemática, ao passo em que seus conceitos são construídos de forma participativa, de modo que os alunos sejam agentes da sua própria aprendizagem.

Para Scheffer (2012, p. 3I), o uso de softwares educacionais “[...], permitem ao estudante, mediado pelo professor, produzir conhecimentos, desenvolver habilidades como resolução de problemas, leitura, imaginação, criatividade e exercício dos conteúdos que já conhece".

Nessa perspectiva, Silva e Pinto (2019), Silva (2018), Nogueira (2018) e Mendes, Jolandek e Pereira (20।8), enfatizam que o uso da Calculadora Gráfica GeoGebra em dispositivos móveis contribui para o ensino e aprendizagem de funções reais. Assim, o objetivo deste artigo é apresentar o levantamento das publicações no Brasil que abordam o uso da Calculadora Gráfica GeoGebra em dispositivos móveis para o ensino de funções reais.

A metodologia utilizada tem uma abordagem qualitativa e o procedimento metodológico se caracteriza como uma revisão bibliográfica no modelo da metassíntese qualitativa, proposto por Matheus (2009), tendo como fonte de dados: o portal de periódicos CAPES, o Scielo e o Google Acadêmico.

Nas seções seguintes deste artigo, contextualizamos a aprendizagem móvel de matemática; em seguida, abordamos alguns aspectos sobre a Calculadora Gráfica GeoGebra; posteriormente apresentamos os procedimentos metodológicos dessa pesquisa e descrevemos os resultados e as discussões e, por fim, as considerações finais. 


\section{APRENDIZAGEM MÓVEL DE MATEMÁTICA}

A propagação do uso de dispositivos móveis na sociedade contemporânea está contribuindo para alterar o processo de ensino aprendizagem no âmbito educacional. Para Prensky (200 I, p. I, tradução nossa'), as mudanças são necessárias, visto que, "[...], os alunos de hoje pensam e processam as informações de modo fundamentalmente diferente dos seus predecessores".

Nessa perspectiva, conforme Carvalho (2017, p. 28), em consonância com a difusão dos dispositivos móveis surge a "aprendizagem móvel, conhecida também por Mobile Learning ou apenas MLearning,". Para a UNESCO (20 I4, p. 08), "a aprendizagem móvel envolve o uso de tecnologias móveis, isoladamente ou em combinação com outras tecnologias de informação e comunicação (TIC), a fim de permitir a aprendizagem a qualquer hora e em qualquer lugar".

Traxler (201 I, apud Carvalho, 2017, p. 32) aponta cinco modos em que a aprendizagem móvel oferece a oportunidade de aprendizagem, a saber:

( I) aprendizagem contingente, em que os alunos podem reagir e responder ao seu ambiente sendo que a aprendizagem e o ensino não são pré-determinados; (2) aprendizagem situada, onde o lugar torna a aprendizagem passível de ser aplicada; (3) aprendizagem autêntica, com atividades relacionadas às metas de aprendizagem imediata; (4) Aprendizagem em contexto, em que a aprendizagem ocorre pela história do ambiente; (5) Aprendizagem personalizada, por meio dos interesses, preferências e habilidades do aprendente.

Conforme menciona Carvalho (20 I 7), a aprendizagem móvel contribui para a educação ser flexível e acessível aos estudantes de diferentes espaços geográficos. Para a UNESCO (20|4, p. 08), a "aprendizagem móvel também abrange esforços em apoio a metas educacionais amplas, como a administração eficaz de sistemas escolares e a melhor comunicação entre escolas e famílias".

No que tange o ensino de matemática, a aprendizagem móvel ainda é uma área de pesquisa emergente, com poucas publicações disponíveis na literatura. Apesar das transformações ocorridas na sociedade nos últimos anos, seus reflexos nos processos educativos relativos à matemática ainda são limitados, (CARVALHO, 2017).

Para Borba et al. (2016, p. 952, tradução nossa²), as pesquisas atuais sobre aprendizagem móvel de matemática podem ser classificadas em três categorias, a saber, " (a) estudos sobre o potencial de dispositivos móveis para o ensino e aprendizagem da matemática; (b) estudos efetivos no uso de dispositivos móveis; e (c) uso de dispositivos móveis na formação de professores de matemática".

De acordo com Carvalho (2017, p.33), "as lacunas nessa área de pesquisa ainda são muitas, apesar das possibilidades que os dispositivos móveis oferecem aos processos educativos de matemática".

\footnotetext{
' [...], todays students think and process information fundamentally differently from their predecessor (PRENSKY, 200 I, p. I).

2 (a) Studies on the potential of mobile devices for teaching and learning mathematics; (b) affective studies on the use of mobile devices; and (c) use of mobile devices in mathematics teacher education (BORBA et al., 20 l6, p. 592).
} 
Borba et al. (2016, 2016, p. 591), ressaltam que:

[.. ] as características dos dispositivos móveis, como a portabilidade, a disponibilidade, o acesso à internet, e sua ampla aceitação entre os jovens e outros, têm feito dos dispositivos móveis um agente emergente capaz de expandir as fronteiras do ensino da matemática e da aprendizagem além das paredes da sala de aula. (tradução nossa ${ }^{3}$ )

Conforme destaca Borba et al. (2016), entendemos que os dispositivos móveis possuem um grande potencial para o ensino aprendizagem de matemática, pois favorece a interação e a cooperação dos estudantes em sala de aula.

De acordo com Sawaya e Putnam (apud Carvalho, 2017, p.34), para utilizar os dispositivos móveis no ensino da matemática, deve-se desenvolver três aspectos, a saber: "os tipos de atividades, as metas de aprendizagem e as potencialidades e possibilidades da tecnologia móvel em questão".

Para Carvalho (2017), os tipos de atividades referem-se ao planejamento instrucional do docente para o uso dos dispositivos móveis em sala de aula. As metas de aprendizagem correspondem aos conteúdos matemáticos e os procedimentos utilizados para os alunos se envolverem com o conteúdo, visando desenvolver a compreensão dos conceitos matemáticos bem como suas aplicações. As potencialidades e possibilidades referem-se aos recursos disponíveis para os dispositivos móveis que serão utilizados nas atividades.

Tendo apresentado alguns aspectos sobre a aprendizagem móvel de matemática, expomos a seguir algumas características da Calculadora Gráfica GeoGebra.

\section{A CALCULADORA GRÁFICA GEOGEBRA}

De acordo com Gonçalves (2016, p. 34), o software GeoGebra foi desenvolvido por Markus Hohenwarter, em sua pesquisa de mestrado no ano de 200 I, junto à Universidade Salzburg, na Áustria. "Sua intenção inicial foi criar um software capaz de lidar de forma dinâmica e simultaneamente com as representações algébricas e geométricas, para os níveis de Ensino Médio e Universitário".

Farias (20|4), afirma que a principal qualidade do GeoGebra, é a possibilidade de interação dinâmica entre diversas áreas da matemática, permitindo que um dado conceito seja compreendido, a partir de sua abordagem na forma visual (gráfica) e algébrica para favorecer a abordagem conceitual.

Xavier (20 I 6), ressalta que o GeoGebra contribui para minimizar as dificuldades encontradas pelos estudantes em alguns conteúdos da disciplina de Matemática pois, facilita o esboço de figuras com pontos, vetores, curvas, parábolas, etc. Além disso, oferece os recursos de que os alunos precisam para resolver

\footnotetext{
3.The characteristics of mobile devices such as portability, availability, access to the internet, and its wide acceptance among young people and others, have made mobile devices an emerging agent capable of expanding the frontiers of mathematics instruction and learning beyond the walls of the classroom (BORBA et al., 2016, p. 59l).
} 
e analisar equações e possibilita estudos com derivadas e representação de funções matemáticas por meio de gráficos.

Nesse sentido, Nogueira (2018), enfatiza que a dinamicidade do GeoGebra presente nas construções de gráficos, figuras e sólidos, permite ao aluno aprimorar a compreensão e o aprofundamento dos conceitos matemáticos.

Gonçalves (20 I6), afirma que no ápice do surgimento do GeoGebra foi disponibilizada uma licença de Código Livre e gratuito, permitindo a vários pesquisadores e profissionais especializados em diversas áreas, contribuirem com o autor do projeto.

A cooperação de vários pesquisadores favoreceu a expansão do software GeoGebra para mais de 190 países, disponível para 68 idiomas, podendo atualmente ser utilizado em computadores e dispositivos móveis (GONÇALVES, 2016).

De acordo com Nogueira (20।8), o GeoGebra para dispositivos móveis foi lançado em 20।3, como uma réplica do software para computadores. No entanto, apenas em 2015, a Calculadora Gráfica GeoGebra foi lançada, permitindo uma flexibilidade no uso de suas ferramentas, principalmente na construção de gráficos.

Nogueira (2018), ressalta que a referida calculadora permite ao usuário esboçar gráficos de funções, resolver equações, encontrar pontos de máximo e mínimo de funções, calcular os zeros de funções, resolver derivadas e integrais, etc.

Para instalar a Calculadora Gráfica GeoGebra, o usuário deve acessar a Google Play, se o dispositivo possuir o sistema operacional Android, ou o iTunes, ou se o dispositivo possuir o sistema operacional iOS. Após a instalação, o ícone do aplicativo irá aparecer na tela principal do dispositivo móvel, para ser utilizado (NOGUEIRA, 20 I8).

Ao inicializar a Calculadora Gráfica GeoGebra, o usuário irá visualizar uma interface contendo as janelas gráfica e algébrica, e as barras de ferramentas e entrada, como ilustra a Figura I. 


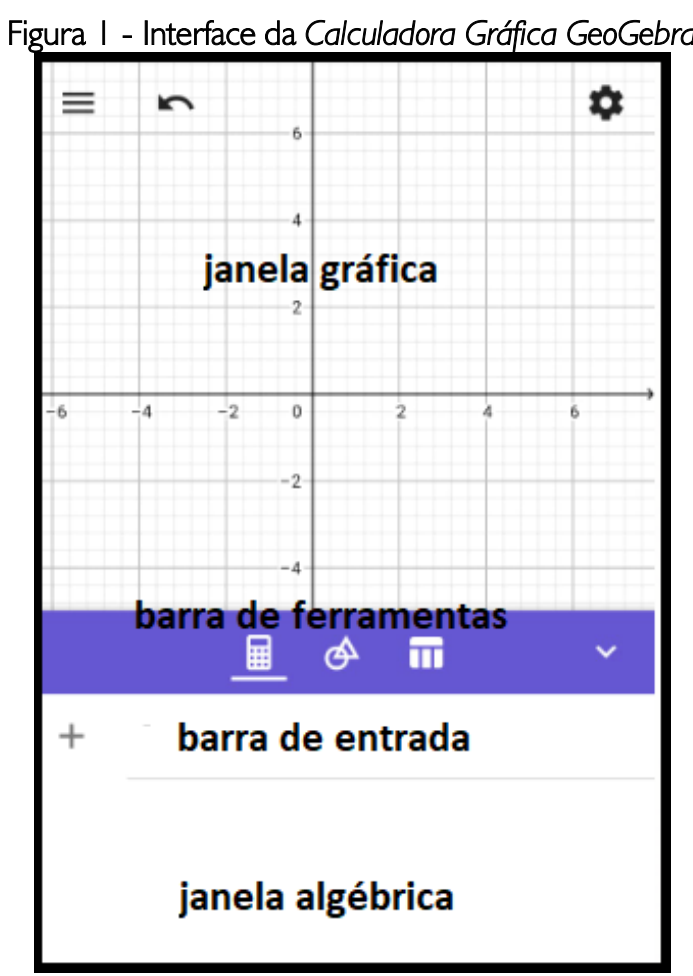

Fonte: elaborado pelos autores.

Tendo apresentado os principais aspectos sobre a Calculadora Gráfica GeoGebra, expomos a seguir, os procedimentos metodológicos adotados nesta investigação.

\section{PROCEDIMENTOS METODOLÓGICOS}

A metodologia adotada nessa pesquisa possui uma abordagem qualitativa. Para Godoy (1995, p. 62), "os estudos denominados qualitativos têm como preocupação fundamental o estudo e a análise do mundo empírico em seu ambiente natural". A autora ressalta que, na pesquisa qualitativa, os dados coletados são usados para obter uma compreensão ampla do fenômeno estudado, considerando que todos os dados da realidade são importantes e devem ser analisados.

A pesquisa caracteriza-se como uma revisão bibliográfica, na perspectiva da metassíntese qualitativa, a qual, segundo Bicudo (20 | 4, p. 09), consiste em uma "investigação pautada em comparações e análises dos dados primários de pesquisas, tomadas como significativas em relação ao tema posto sob foco".

Para Bicudo (idem), o objetivo da metassíntese qualitativa é "desenvolver um conhecimento teórico que apresente um nível de abstração mais elevado, em relação às sínteses interpretativas dos estudos primários, visando à maior possibilidade de aplicações em situações práticas".

De acordo com Alencar e Almouloud (2017), a metassíntese qualitativa é uma metodologia proveniente de pesquisas internacionais, sendo muito utilizada na área da saúde. As pesquisas em outras áreas surgiram em 1998, pela Fundação Cochrane, que estabeleceu o "Qualitative Research Methods 
Working Group", que fornece orientações metodológicas a pesquisadores que usam as revisões sistemáticas conforme os padrões definidos pela instituição.

Nessa perspectiva, Matheus (2009) enfatiza segundo as orientações da Fundação Cochrane, que a metassíntese qualitativa é desenvolvida em seis etapas, a saber: I) identificar a importância intelectual e o objetivo da pesquisa; 2) decidir o que é relevante aos interesses e, consequentemente, os critérios iniciais de inclusão dos estudos; 3) fazer a leitura dos estudos; 4) determinar como os estudos estão relacionados; 5) formar novas afirmações; e 6) organizar a nova explicação, de forma que seja equivalente a todos os estudos pesquisados. Assim, ressaltamos que a presente pesquisa foi elaborada com base nas etapas explicitadas anteriormente.

As bases de dados utilizadas foram, o portal de periódicos CAPES, o SCIELO e o Google Acadêmico, sendo analisadas as produções desenvolvidas no período entre 2013 a 2019 , devido ao fato de o aplicativo do software GeoGebra para dispositivos móveis ter sido lançado em 2013.

Tendo apresentado os principais aspectos sobre o procedimento metodológico, expomos a seguir, os resultados da pesquisa nas bases de dados.

\section{$5 \quad$ RESULTADOS E DISCUSSÕES}

Para selecionar os trabalhos no portal de periódicos da CAPES, as buscas textuais foram realizadas entre aspas duplas, pois com essa técnica as pesquisas contemplam exatamente o descritor desejado. A Figura 2, ilustra os descritores e a quantidade de trabalhos encontrados nas três buscas realizadas.

Figura 2 - Resultados das três buscas no portal de periódicos CAPES

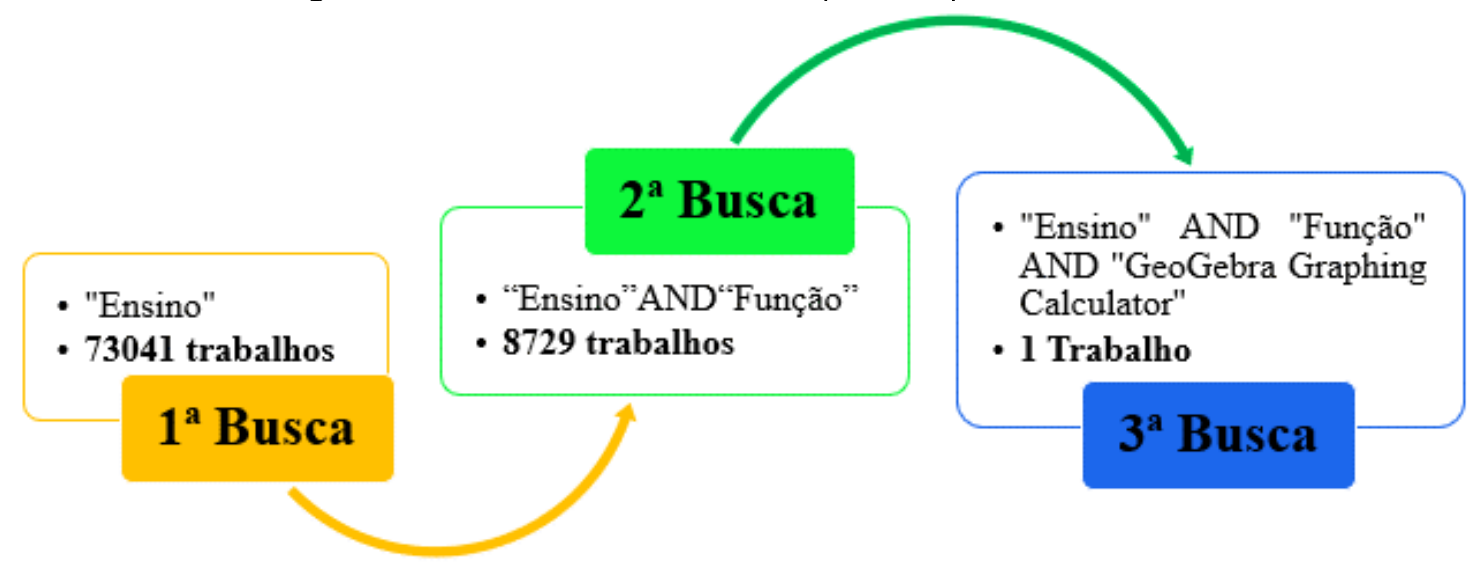

Fonte: elaborado pelos autores.

Conforme ilustra a Figura I, na primeira busca, utilizamos o descritor "Ensino", e foram encontrados 7304 I trabalhos. Considerando que o descritor ensino contempla várias áreas do conhecimento, na segunda busca fizemos um refinamento dos dados, por meio da combinação de 
descritores com a utilização de operadores de buscas. Assim, utilizando a combinação "Ensino" AND "Função", foram encontrados 8729 trabalhos. Na terceira busca, fizemos novamente um recorte dos dados, utilizando a combinação de descritores, visto que o foco da pesquisa são os trabalhos que abordam o ensino de funções reais com a Calculadora Gráfica GeoGebra. Assim, utilizando a combinação "Ensino" AND "Função" AND "GeoGebra Graphing Calculator ", foi encontrado somente um trabalho.

$\mathrm{Na}$ pesquisa realizada na base de dados da SCIELO, utilizamos também a combinação de descritores com os operadores de buscas. Utilizando a combinação, "Ensino and Função and GeoGebra Graphing Calculator", não encontramos nenhum trabalho relacionado com a temática da pesquisa.

Considerando a baixa quantidade de trabalhos nas bases de dados pesquisadas anteriormente, foram realizadas buscas no Google Acadêmico. Na busca utilizamos a frase "Ensino de funções com a Calculadora Gráfica GeoGebra" e foram encontrados 1450 trabalhos publicados no período de 2013 a 2019. No entanto, após a leitura dos títulos e resumos dos trabalhos encontrados, foram excluídos os trabalhos repetidos e as produções não relacionadas como o uso da Calculadora Gráfica GeoGebra em dispositivos móveis para o ensino de funções. Após esses procedimentos, selecionamos 4 trabalhos para realizarmos a leitura completa e análise, conforme descritos no Quadro I.

Quadro I - Trabalhos selecionados na base de dados do Portal de Periódicos CAPES e Google Acadêmico

\begin{tabular}{|l|c|c|c|c|}
\hline \multicolumn{1}{|c|}{ Título } & Autores & Ano & Tipo de publicação & Base de dados \\
\hline $\begin{array}{l}\text { Funções quadráticas e tecnologias } \\
\text { móveis: ações cooperativas em um } \\
\text { experimento no ensino médio }\end{array}$ & $\begin{array}{c}\text { Rodrigo Sychocki da Silva; } \\
\text { Shéridan dos Reis Pinto }\end{array}$ & 2019 & Artigo & Portal de \\
\hline $\begin{array}{l}\text { Uma abordagem com mobile } \\
\text { learning: ensino de funções afins } \\
\text { auxiliado pelo GeoGebra }\end{array}$ & $\begin{array}{c}\text { Luiz Otavio Rodrigues } \\
\text { Mendes; Emilly Gonzales } \\
\text { Jolandek; Ana Lúcia Pereira }\end{array}$ & 2018 & Artigo \\
\hline $\begin{array}{l}\text { O uso da Calculadora Gráfica } \\
\text { GeoGebra no smartphone como } \\
\text { ferramenta para o ensino das } \\
\text { funções exponencial e logarítmica }\end{array}$ & $\begin{array}{c}\text { Eduardo Leandro Peres } \\
\text { Nogueira }\end{array}$ & Doogle Acadêmico \\
\hline $\begin{array}{l}\text { A utilização do aplicativo GeoGebra } \\
\text { para smatphone como recurso } \\
\text { didático nas aulas de matemática no } \\
\text { Ensino Fundamental }\end{array}$ & $\begin{array}{c}\text { Elainny Roma Pereira da } \\
\text { Silva }\end{array}$ & 2018 & Google Acadêmico \\
\hline
\end{tabular}

Fonte: elaborado pelos autores.

Silva e Pinto (2019, p. I10) investigaram "como as ações cooperativas entre os estudantes influencia na aprendizagem das funções quadráticas".

A metodologia utilizada é de cunho qualitativo, sendo os sujeitos da pesquisa alunos do $1^{\circ}$ ano do ensino médio, do colégio de aplicação da Universidade Federal do Rio Grande do Sul (UFRGS).

A coleta de dados foi realizada, por meio da gravação de áudios e registros dos alunos, durante a aplicação de atividades sobre a variação de parâmetros da função quadrática, utilizando software GeoGebra Graphing Calculator (SILVA; PINTO, 2019). Os autores ressaltam que, cada questão utilizada nas 
atividades, foi construída com o intuito de provocar, a partir do trabalho coletivo, as reflexões dos participantes, buscando a construção de uma resposta para a pergunta central da pesquisa.

De acordo com Silva e Pinto (2019), ao utilizar a calculadora gráfica do GeoGebra notou-se que o "movimento" fornecido aos elementos predominantemente estáticos nos livros didáticos oportunizou aos estudantes conduzir o processo da própria aprendizagem, ou seja, a partir de suas palavras elaboram os próprios entendimentos sobre a temática que estão estudando.

Mendes, Jolandek e Pereira (2018, p. 43) apresentaram "uma proposta de ensino que utiliza o Mobile Learning como uma metodologia para o Ensino de Matemática, para o conteúdo função afim no Ensino Médio, utilizando o aplicativo matemático GeoGebra em Smartphones e Tablets".

A metodologia utilizada possui uma abordagem qualitativa, caracterizando-se como uma pesquisa bibliográfica no modelo da revisão sistemática proposto por Sampaio e Mancini (2007).

A proposta de ensino a partir da utilização do aplicativo Calculadora Gráfica-GeoGebra , foi organizada em cinco etapas sendo utilizada a estratégia do Bring your own device (BYOD), com o intuito de trabalhar alguns conceitos da função afim, a saber: Zero da função; Coeficiente Angular; Coeficiente Linear; Sinal da função; função crescente e decrescente. Essa prática consiste, segundo Mendes; Jolank; Pereira (2018, p. 44) "em cada indivíduo utilizar o seu dispositivo móvel, smartphones ou tablets".

Para os autores (idem, p. 57), a proposta "apresentada pode ser uma possibilidade diferenciada para se trabalhar o conteúdo função afim na Matemática, bem como outros conteúdos que possam ser contemplados".

Nogueira (2018, p. 09) investigou "o uso da Calculadora Gráfica-GeoGebra no celular smartphone como ferramenta para o ensino das Funções Exponencial e Logarítmica".

A metodologia utilizada possui uma abordagem quali-quantitativa, sendo os sujeitos da pesquisa alunos de cinco turmas de $1^{\circ}$ ano do Ensino Médio da Escola Estadual Professor Anísio Teixeira, situada em Natal, no estado do Rio Grande do Norte.

A coleta de dados foi realizada por meio das respostas dos alunos, obtidas durante a aplicação de duas atividades como o intuito de verificar a influência de parâmetros no comportamento dos gráficos das funções exponencial e logarítmica, utilizando a Calculadora Gráfica GeoGebra. Além disso, utilizou-se um questionário após a aplicação das atividades, para avaliar o trabalho desenvolvido com as turmas, bem como os benefícios da utilização da Calculadora Gráfica GeoGebra, na resolução das atividades.

De acordo com Nogueira (20 I8), por meio das respostas obtidas nas atividades e as opiniões dos alunos foi possível constatar que a referida calculadora facilitou a compreensão do conteúdo trabalhado, além de aumentar o interesse dos alunos, sendo um recurso de grande potencial para o ensino aprendizagem da matemática. 
Silva (20 I 8) apresentou em sua pesquisa um levantamento do perfil de professores de matemática quanto à utilização das mídias digitais móveis, comparando-a com o uso dos computadores. Além disso, o autor analisou o perfil dos alunos quanto à utilização dos smartphones como ferramenta de estudos e recurso didático em sala de aula, analisando a eficiência do aprendizado por meio da calculadora gráfica GeoGebra.

A metodologia utilizada possui uma abordagem quali-quantitativa, sendo caracterizada como uma pesquisa descritiva. Os sujeitos da pesquisa foram 13 alunos do $9^{\circ}$ ano Ensino Fundamental da Escola Municipal de São José de Ribamar, e 35 professores da rede pública de ensino do estado do Maranhão.

Para analisar o perfil dos alunos, quanto ao uso das tecnologias no dia-a-dia, o autor aplicou um questionário, posteriormente utilizou a Calculadora Gráfica GeoGebra para o estudo das funções polinomiais do $1^{\circ}$ e $2^{\circ}$ grau. Em relação aos professores foi aplicado um questionário online viabilizando a análise do perfil de cada participante.

Com base nos questionários, segundo o autor, foi possível constatar que os professores de matemática que participaram da pesquisa não estão acompanhado o desenvolvimento tecnológico voltado para o ensino aprendizagem da matemática. Além disso, foi possível perceber que a Calculadora Gráfica GeoGebra contribui para o ensino e aprendizagem das funções polinomiais do $1^{\circ}$ e $2^{\circ}$ grau.

Após a análise dos trabalhos selecionados, constatamos que, apesar de serem pesquisas distintas, os objetivos de Silva e Pinto (2019), Nogueira (20 I 8) e Silva (20 I8), possuem características semelhantes, pois têm, a finalidade de analisar o uso da Calculadora Gráfica GeoGebra como recurso didático para o ensino de funções reais, no entanto o objetivo de Mendes, Jolandek e Pereira (20। 8), difere dos demais pois, visa a apresentar uma proposta de ensino mediado pela Calculadora Gráfica GeoGebra .

Em relação aos sujeitos e às funções reais abordadas nas pesquisas, Silva e Pinto (20।9), Nogueira (2018) e Silva (2018), apesar de trabalharem com alunos do $1^{\circ}$ do Ensino Médio, apresentaram abordagens distintas, ou seja, Silva e Pinto (2019), trabalharam atividades sobre função quadrática, Nogueira (20 I 8) trabalhou atividades sobre as funções exponencial e logarítmica, e Silva (20 I 8), trabalhou atividades sobre as funções afim e quadrática. Por outro lado, Mendes, Jolandek e Pereira (20l8), diferentemente dos demais, apresentaram uma proposta para trabalhar com os alunos do ensino médio, atividades sobre a função afim.

Quanto à metodologia, Silva e Pinto (2019) e Mendes, Jolandek e Pereira (20I8), utilizaram uma abordagem qualitativa, Nogueira (20 I8) e Silva (20 I 8), utilizaram uma abordagem quali-quantitativa.

Na coleta de dados, Silva e Pinto (2019) utilizaram a gravação de áudios e atividades discursivas sobre os seguintes estudos da função quadrática, a saber: variação de parâmetros; zeros da função e pontos de máximos e mínimos. Nogueira (20|8), utilizou duas atividades sobre a variação de parâmetros das funções exponencial e logarítmica enquanto Silva (20।8), utilizou questionários e atividades discursivas 
sobre os seguintes estudos das funções afim e quadrática, a saber: variação dos coeficientes; construção de gráficos, classificação de função crescente e decrescente; vértice da parábola e concavidade da parábola.

Em síntese, percebemos que, apesar de utilizarem abordagens metodológicas diferentes, os resultados das pesquisas convergiram, no sentido de que, a Calculadora Gráfica GeoGebra, contribui na aprendizagem de funções reais, em virtude do recurso de visualização gráfica, com isso estimula a participação e a cooperação dos estudantes nas aulas de matemática, de modo que os mesmos sejam construtores de suas próprias aprendizagens.

\section{CONSIDERAÇÕES FINAIS}

Esta pesquisa teve a finalidade de apresentar um panorama das pesquisas nacionais que abordam o uso da Calculadora Gráfica GeoGebra em dispositivos móveis, para o ensino de funções reais.

As pesquisas analisadas apontaram que a Calculadora Gráfica GeoGebra contribui para o ensino aprendizagem de funções reais, pois facilita a visualização gráfica, estimula a participação e a cooperação dos estudantes nas aulas de matemática, de modo que os mesmos sejam construtores das suas próprias aprendizagens. No entanto, cabe ressaltar que há poucos trabalhos disponíveis na literatura com esta temática, constituindo assim uma área emergente a novas pesquisas.

O desenvolvimento deste estudo ampliou a discussão sobre o uso de dispositivos móveis como recurso pedagógico nas aulas de matemática, apontando contribuições e limitações das pesquisas analisadas.

Espera-se que esse estudo possa contribuir com o desenvolvimento de novas pesquisas com a temática investigada, tendo em vista que a Calculadora Gráfica GeoGebra possui várias funcionalidades que podem ser utilizadas em sala de aula para trabalhar vários conteúdos matemáticos.

\section{REFERÊNCIAS}

ALENCAR, Edvonete S.; ALMOULOUD, Saddo Ag. A metodologia de pesquisa: metassíntese qualitativa. Reflexão e Ação, v. 25, n. 3, p. 204-220, 2017. Diponivel em:

https://online.unisc.br/seer/index.php/reflex/article/view/973I. Acesso em: 01 de mai. 2020.

BICUDO, Maria A. V. Meta-análise: seu significado para a pesquisa qualitativa. Revemat: revista eletrônica de educação matemática, v. 9, p. 7-20, 20।4. Disponível em: https://periodicos.ufsc.br/index.php/revemat/article/view/I98I - 1322.20 I4v9nespp7. Acesso em: 0 I de mai. 2020.

Borba, Marcelo C. et al. Blended learning, e-learning and mobile learning in mathematics education. ZDM - Mathematics Education, v. 48, n. 5, p. 589-610, 2016. Disponível em: http://hdl.handle.net/I I449//68802. Acesso em: 10 jun. 2020. 
INSTITUTE GEOGEBRA INTERNATIONAL. Calculadora Gráfica-Geogebra: dynamic mathematics for schools. Version: 6.0.590.0-wgraphing. Salzburg: Department of Mathematics, University of Salzburg, 2020. Disponível em: https://www.geogebra.org/download. Acesso em: 20 jun. 2020.

CARVALHO, Jaqueline N. O Papel dos Dispositivos Móveis para a Aprendizagem Móvel de Matemática. Dissertação (Mestrado) - Universidade do Estado de Mato Grosso, Barra do Bugres. 2017. Disponível em: http://portal.unemat.br/media/files/JA

QUELINE_NUNES_CARVALHO.pdf. Acesso em: 29 de abr. de 2020.

CROMPTON, Helen; TRAXLER, John (Eds.). Mobile learning and mathematics: foundations, design and case studies. Florence Routledge, New York, 2015.

FARIAS, Maria M. do R. Introdução a noções de cálculo diferencial e integral no ensino médio no contexto das TIC: implicações para prática do professor que ensina matemática. Tese (Doutorado). Instituto de Geociências e Ciências Exatas, Universidade Estadual Paulista (UNESP), Rio Claro - SP, 20 I4. Disponível em: https://repositorio.unesp.br/handle// I 449/I32207. Acesso em: 16 fev. 2020.

GODOY, Arlinda S. Introdução à pesquisa qualitativa e suas possibilidades: Uma revisão histórica dos principais autores e obras que refletem esta metodologia de pesquisa em Ciências Sociais. Revista de Administração de Empresas, p. 57-63, 1995. Disponível em:

http://bibliotecadigital.fgv.br/ojs/index.php/rae/article/viewFile/38183/36927 . Acesso em: 30 de abr. de 2019.

GONÇALVES, William. V. O Transitar entre a Matemática do Matemático, a Matemática da Escola e a Matemática do GeoGebra: Um Estudo de como Professores de Matemática lidam com as possibilidades e limitações do GeoGebra. Tese (Doutorado) - Faculdade de Ciências, Universidade Estadual Paulista (UNESP), Bauru - SP, 20 I6. Disponível em: http://hdl.handle.net/I I 449/I4395 I. Acesso em: 0 I jan. 2020.

LIMA, Patrícia R. B. de; FALKEMBACH, Gilse A. M.; TAROUCO, Liane M. R.. Objetos de aprendizagem no contexto de m-learning. Objetos de aprendizagem: teoria e prática. Porto Alegre: Evangraf, 2014. p. 431 - 447, 2014. Disponível em: https://www .lume.ufrgs.br/bitstream/handle/I 0 I 83// 93565/00093724 I .pdf?sequence= I \&isAllowed=y. Acesso em 10 de mai. de 2020.

MATHEUS, Maria C. C. Metassíntese qualitativa: desenvolvimento e contribuições para a prática baseada em evidências. Acta paulista de enfermagem, v. 22, n. spe2, p. 543-545, 2009. Disponível em: https://www.redalyc.org/pdf/3070/307023853019.p df. Acesso em: 10 mai. de 2020.

MENDES, Luiz O. R.; JOLANDEK, Emilly G.; PEREIRA, Ana L. Uma abordagem com mobile learning: ensino de funções afım auxiliado pelo GeoGebra. International Journal of Knowledge Engineering and Management (IJKEM), v. 7, n. 19, p. 4I-58, 2018. Disponível em: http://stat.ijie.incubadora.ufsc.br/index.php/IJKEM/article/view/5242. Acesso em I de mai. 2020.

NOGUEIRA, Eduardo L. P. O uso da calculadora gráfica GeoGebra no smartphone como ferramenta para o ensino das funções exponencial e logarítmica. Dissertação ((Mestrado) - Universidade Federal do Rio Grande do Norte, Natal - RN, 20 8. Disponível em: http://bdtd.ibict.br/vufind/Record/UFRN_445e0e8cc3793aba9bbd97d I d53d6770. Acesso em: I 2 de mai. 2020. 
PACHECO, José Adson D.; BARROS, Janaina $\vee$. O uso de softwares educativos no ensino de matemática. Revista Diálogos, v. 8, p. 5-13, 2013. Disponível em:

http://www.revistadialogos.com.br/Dialogos_8/Adson_Janaina.pdf. Acesso em 9 de mai. de 2020.

Prensky, Marc. Digital Natives, Digital Immigrants Part I, On the Horizon, v. 9, n. 5, p. I-6, 200 I.

Disponível em: https://doi.org/I0.1 I08/I 0748 I20 I I04248 I6. Acesso em: 28 de abr. 2020.

SCHEFFER, Nilce F. A argumentação em matemática na interação com tecnologias. Ciência e Natura, v. 34, n. I, p. 23-38, 2012. Disponível em: https://periodicos.ufsm.br/cienciaenatura/article/view/9352. Acesso em: 09 de dez. de 2020.

SILVA, Rodrigo S. da; PINTO, Shéridan dos R. Funções quadráticas e tecnologías móveis: ações cooperativas em um experimento no ensino médio. Góndola, Enseñanza y Aprendizaje de las Ciencias: Góndola, Ens Aprend Cienc, v. I4, n. I, p. 108-125, 2019. Disponível em:https://revistas.udistrital.edu.co/ojs/Index.php/GDLA/article/view/I33 17/pdf. Acesso em 8 de abr. de 2020.

SILVA, Elanny R. P. A utilização do aplicativo Geogebra para smartphone como recurso didático nas aulas de matemática do Ensino Fundamental. Dissertação (Mestrado) - Universidade Federal do Maranhão, São Luiz - MA, 2018. Disponível em:

https:/tedebc.ufma.br/jspui/bitstream/tede/2555/2/ELANNYSILVA.pdf . Acesso em I0 de mai. de 2019.

UNESCO, Organização das Nações Unidas para a Educação, Ciência e Cultura. Diretrizes de políticas para a aprendizagem móvel, 2014. Disponível em:

http://unesdoc.unesco.org/images/0022/002277/227770por.pdf. Acesso em: 25 abr. 2020.

XAVIER, Rafael G. Geometria espacial - um curso com Geogebra. Dissertação (Mestrado) Universidade Federal de Goiás, Goiânia - GO, 2016. Disponível em :

https://repositorio.bc.ufg.br/tede/bitstream/tede/6696/5/Disserta\%C3\%A7\%C3\%A3o

\%20-\%20Rafael\%20Gomes\%20Xavier\%20-\%2020 I6.pdf . Acesso em 10 de maio. 2020.

\section{COMO CITAR ESSE ARTIGO}

\section{Associação Brasileira de Normas Técnicas (ABNT)}

CARVALHO, Joilson Ferreira de; SOUTO, Daise Lago Pereira; GONÇALVES, William Vieira. O uso da calculadora gráfica GeoGebra em dispositivos móveis para o ensino de funções reais: um olhar para as publicações no Brasil. Debates em Educação, Maceió, v. 12, p. 315-327, dez. 2020. ISSN 2175-6600. Disponível em: https://www.seer.ufal.br/index.php/debateseducacao/article/view/l 0464. Acesso em: dd $\mathrm{mmm}$. aaaa.

\section{American Psychological Association (APA)}

Carvalho, J., Souto, D., \& Gonçalves, W. (2020). O uso da calculadora gráfica GeoGebra em dispositivos móveis para o ensino de funções reais: um olhar para as publicações no Brasil. Debates em Educação, 12(Esp2), 3 I5-327. doi: https://doi.org/10.28998/2 I 75-6600.2020v12nEsp2p3 I5-327 